\title{
NON-COMPACT LAMINATION CONVEX HULLS
}

\section{ENVELOPPE LAMINEUSEMENT CONVEXE NON COMPACT}

\author{
Jan KOLÁ:̌ ${ }^{1}$ \\ Dept. Math. Anal., Charles University, Sokolovská 83, 18675 Praha 8, Czech Republic \\ Received 31 July 2001 \\ Dedicated to my friends Renáta and Ivan Zahrádka
}

\begin{abstract}
For $K$ a compact set of $m \times n$ matrices, let $\mathrm{L}(K)$ denote the lamination convex hull of $K$.

We give an example of a compact set $K$ of symmetric two by two matrices such that $\mathrm{L}(K)$ is not compact, and similar examples for separate convexity in $\mathbb{R}^{3}$ and bi-convexity in $\mathbb{R}^{2} \times \mathbb{R}$. Furthermore we show that function $\tilde{\mathrm{L}}$, where $\tilde{\mathrm{L}}(K)=\overline{\mathrm{L}(K)}$, is not upper semi-continuous with respect to Hausdorff metric on the space of all compact sets $K$ of diagonal $3 \times 3$ matrices.
\end{abstract}

(C) 2003 L'Association Publications de l'Institut Henri Poincaré. Published by Elsevier B.V. All rights reserved

MSC: 26B25; 52A30

Keywords: Lamination convex hull; Bi-convexity; Separate convexity; Rank-one convexity

RÉSUMÉ. - Si $K$ est un ensemble compact des matrices du type $m \times n, \mathrm{~L}(K)$ signifie le plus petit ensemble lamineusement convexe contenant $K$. (Un ensemble $K$ est lamineusement convexe si $[a, b] \subset K$ pour tous $a, b \in K$ tels que $a-b$ est une matrice de rang 1.)

Nous démontrons qu'il y a $K$, un ensemble compact des matrices symétriques d'ordre 2 tel que $\mathrm{L}(K)$ ne soit pas compact. Nous présentons aussi des exemples similaires pour convexité séparée dans $\mathbb{R}^{3}$ et bi-convexité dans $\mathbb{R}^{2} \times \mathbb{R}$. En plus, nous démontrons que l'application $\tilde{\mathrm{L}}: K \mapsto \overline{\mathrm{L}(K)}$ n'est pas semi-continue superieurement sur l'espace des ensembles compacts de matrices diagonales d'ordre 3 muni de la métrique de Hausdorff.

(c) 2003 L'Association Publications de l'Institut Henri Poincaré. Published by Elsevier B.V. All rights reserved

Mots Clés: Enveloppe lamineusement convexe; Bi-convexité; Convexité separée; Enveloppe convexe de rang un

E-mail address: kolar@karlin.mff.cuni.cz (J. Koláŕ).

${ }^{1}$ Research was partially supported by Max Planck Institute for Mathematics in the Sciences in Leipzig. 


\section{Introduction}

We denote by $\mathbb{M}^{m \times n}$ the set of all real $m \times n$ matrices with the $\mathbb{R}^{m n}$ norm; $\mathbb{M}_{\text {sym }}^{n \times n}, \mathbb{M}_{\text {diag }}^{n \times n}$ are the sets of symmetric and diagonal $n \times n$ matrices, respectively. A set $K \subset \mathbb{M}^{m \times n}$ is called lamination convex [4] if for all $A, B \in K$, which satisfy $\operatorname{rank}(A-B)=1$, one has $(1-\lambda) A+\lambda B \in K$ for all $\lambda \in(0,1)$. For a given $K \subset \mathbb{M}^{m \times n}$, the lamination convex hull $\mathrm{L}(K)$ is defined as the smallest lamination convex set which contains $K$ [4].

Zhang [6] writes that "it is not clear in general whether for a compact set, the lamination convex hull is closed". In fact, it is easy to obtain a counter-example in $\mathbb{M}^{2 \times 4}$ from a paper of Aumann and Hart [1], see Example 2.4. The main purpose of this paper is to give an example of a compact set $K \subset \mathbb{M}_{\text {sym }}^{2 \times 2}$ such that $\mathrm{L}(K)$ is not compact.

For convenience, we identify $\mathbb{M}_{\text {sym }}^{2 \times 2}$ with $\mathbb{R}^{3}$ by the linear bijection $\phi(x, y, z)=$ $\left(\begin{array}{cc}z+x & y \\ y & z^{-x}\end{array}\right)$. We say that $(x, y, z) \in \mathbb{R}^{3}$ is a rank-one direction if $\operatorname{det} \phi(x, y, z)=$ $z^{2}-x^{2}-y^{2}=0$, that points $A, B$ are rank-one connected if $B-A$ is a rank-one direction and that a set $K \subset \mathbb{R}^{3}$ is lamination convex if $(1-\lambda) A+\lambda B \in K$ whenever $A, B \in K$ are rank-one connected and $\lambda \in(0,1)$. Again, the lamination convex hull $\mathrm{L}(K)$ of a set $K \subset \mathbb{R}^{3}$ is the smallest lamination convex set containing $K$. Obviously, $K \subset \mathbb{R}^{3}$ is lamination convex if and only if $\phi(K) \subset \mathbb{M}_{\text {sym }}^{2 \times 2}$ is lamination convex, and $\mathrm{L}(\phi(K))=\phi(\mathrm{L}(K))$ for every $K \subset \mathbb{R}^{3}$.

THEOREM 1.1. - There is a compact set $K \subset \mathbb{M}_{\text {sym }}^{2 \times 2}$ such that $\mathrm{L}(K)$ is not compact.

Before proving the theorem for the symmetric two by two matrices in Section 3 we would like to consider the easier case of $\mathbb{M}^{m \times n}$ with $\max (m, n)>2$ where examples can be constructed using related notions of separate convexity and bi-convexity. In Section 4 we explain consequences to upper semi-continuity of the mapping $K \mapsto \mathrm{L}(K)$.

\section{Examples}

The diagonal matrix $\left(\begin{array}{lll}x & 0 & 0 \\ 0 & y & 0 \\ 0 & 0 & z\end{array}\right)$ is of rank one if and only if exactly one of the numbers $x, y, z$ is non-zero. Let us say that $K \subset R^{n}$ is separately lamination convex if $K$ contains every segment with end-points in $K$ which is parallel to one of the coordinate axes. This is equivalent to lamination convexity of the corresponding set of diagonal matrices. The separately lamination convex hull $\mathrm{L}_{\mathrm{sc}}(K)$ is defined to be the smallest separately lamination convex set in $\mathbb{R}^{n}$ that contains $K$.

Example 2.1 (Separate convexity in $\mathbb{R}^{3}$ and diagonal $3 \times 3$ matrices). - Let

$$
\begin{aligned}
K= & \{(1,1,1)\} \cup\{(-1,0,0),(0,-1,0),(0,0,-1)\} \\
& \cup \bigcup_{n \in \mathbb{N}}\left\{\left(-1, \frac{1}{n}, \frac{1}{n}\right),\left(\frac{1}{n+1},-1, \frac{1}{n}\right),\left(\frac{1}{n+1}, \frac{1}{n+1},-1\right)\right\} .
\end{aligned}
$$

By induction, $\mathrm{L}_{\mathrm{sc}}(K)$ contains each of the segments

$$
\left[\left(\frac{1}{n}, \frac{1}{n}, \frac{1}{n}\right),\left(-1, \frac{1}{n}, \frac{1}{n}\right)\right] \ni\left(\frac{1}{n+1}, \frac{1}{n}, \frac{1}{n}\right),
$$




$$
\begin{aligned}
& {\left[\left(\frac{1}{n+1}, \frac{1}{n}, \frac{1}{n}\right),\left(\frac{1}{n+1},-1, \frac{1}{n}\right)\right] \ni\left(\frac{1}{n+1}, \frac{1}{n+1}, \frac{1}{n}\right),} \\
& {\left[\left(\frac{1}{n+1}, \frac{1}{n+1}, \frac{1}{n}\right),\left(\frac{1}{n+1}, \frac{1}{n+1},-1\right)\right] \ni\left(\frac{1}{n+1}, \frac{1}{n+1}, \frac{1}{n+1}\right)}
\end{aligned}
$$

for every $n \in \mathbb{N}$. Consequently, $(0,0,0)$ belongs to the closure of $\mathrm{L}_{\mathrm{sc}}(K)$. On the other hand, it does not belong to $\mathrm{L}_{\mathrm{sc}}(K)$ since the set

$$
\begin{aligned}
& \{(-1,0,0),(0,-1,0),(0,0,-1)\} \\
& \quad \cup\left\{A \in \mathbb{R}^{3}: \text { at least two coordinates of } A \text { are strictly positive }\right\}
\end{aligned}
$$

is separately lamination convex and contains $K$. Thus $K \subset \mathbb{R}^{3}$ is compact, but $\mathrm{L}_{\mathrm{sc}}(K)$ is not and the same is true for the lamination convex hull of the compact set

$$
\left\{\left(\begin{array}{lll}
x & 0 & 0 \\
0 & y & 0 \\
0 & 0 & z
\end{array}\right):(x, y, z) \in K\right\} .
$$

Example 2.2 (Separate convexity in $\mathbb{R}^{2}$ and diagonal $2 \times 2$ matrices). - The lamination convex hull of a compact subset of $\mathbb{M}_{\text {diag }}^{2 \times 2}$ is always compact. This follows by the next result which is due to Kirchheim [3].

PROPOSITION 2.3. - If $C \subset \mathbb{R}^{2}$ is compact, then $\mathrm{L}_{\mathrm{sc}}(C)$ is compact as well.

Proof (B. Kirchheim). - By $x_{1}, x_{2}$ we denote the two coordinates of $x \in \mathbb{R}^{2}$, and $e_{1}=(1,0), e_{2}=(0,1)$. Let $\mathrm{L}_{\mathrm{sc}}^{(0)}(C)=C$ and for $k \in N$ let

$$
\mathrm{L}_{\mathrm{sc}}^{(k)}(C)=\bigcup\left\{[y, z]: y, z \in \mathrm{L}_{\mathrm{sc}}^{(k-1)}(C), y_{1}=z_{1} \text { or } y_{2}=z_{2}\right\} .
$$

Then $\mathrm{L}_{\mathrm{sc}}^{(k)}(C)$ are compact and $\mathrm{L}_{\mathrm{sc}}(C)=\bigcup_{k} \mathrm{~L}_{\mathrm{sc}}^{(k)}(C)$. We say that $\operatorname{gen}_{C}(x)=k$ provided $x \in \mathrm{L}_{\mathrm{sc}}^{(k)}(C) \backslash \mathrm{L}_{\mathrm{sc}}^{(k-1)}(C)$. Suppose the claim fails. Then we can find a compact set $C \subset \mathbb{R}^{2} \backslash[-1,1]^{2}$ such that

$$
0 \in \overline{\mathrm{L}_{\mathrm{sc}}(C)} \backslash \mathrm{L}_{\mathrm{sc}}(C) .
$$

Obviously, for $i=1,2$ we find $\sigma_{i} \in\{-1,1\}$ such that

$$
t \cdot \sigma_{i} e_{i} \notin \mathrm{L}_{\mathrm{sc}}(C) \quad \text { whenever } t \geqslant 0 .
$$

Moreover, we find $\varepsilon>0$ such that

$$
\sigma_{i} x_{i}<-\varepsilon \quad \text { or } \quad\left|x_{3-i}\right|>\varepsilon \quad \text { for all } x \in C, i \in\{1,2\} .
$$

Now we set

$$
M_{i}=\left\{x:\left|x_{3-i}\right| \leqslant \varepsilon \text { and } \sigma_{i} x_{i} \geqslant-\varepsilon\right\}, \quad M_{i}^{+}=\left\{x \in M_{i}: \sigma_{i} x_{i} \geqslant 0\right\}
$$

and claim that

$$
\mathrm{L}_{\mathrm{sc}}(C) \cap M_{i}^{+}=\emptyset
$$


Let us assume that (4) is not true for an $i \in\{1,2\}$. Let $g=\min \left\{\operatorname{gen}_{C}(x): x \in \mathrm{L}_{\mathrm{sc}}(C) \cap\right.$ $M_{i}^{+}$\}. Due to (3) we know $g \geqslant 1$ and find $x$ in the compact set $M_{i}^{+} \cap \mathrm{L}_{\mathrm{sc}}^{(g)}(C)$ maximizing the non-negative function $x \mapsto \sigma_{i} x_{i}$ over this set. By the definition of $\mathrm{L}_{\mathrm{sc}}^{(g)}(C)$ there are $y, z \in \mathrm{L}_{\mathrm{sc}}^{(g-1)}(C)$ such that $x \in \mathrm{L}_{\mathrm{sc}}^{(1)}(\{y, z\})$. From the maximality of $\sigma_{i} x_{i}$ we conclude that $\sigma_{i} y_{i}=\sigma_{i} z_{i}=\sigma_{i} x_{i} \geqslant 0$. The definition of $g$ implies that $y, z \notin M_{i}^{+}$, hence $\left|y_{3-i}\right|,\left|z_{3-i}\right|>$ $\varepsilon$ and $y_{3-i} z_{3-i}<0$. Consequently,

$$
\mathrm{L}_{\mathrm{sc}}(C) \cap\left\{t \cdot \sigma_{i} e_{i}: t \geqslant 0\right\} \supset[y, z] \cap\left\{t \cdot \sigma_{i} e_{i}: t \geqslant 0\right\}=\left\{x_{i} e_{i}\right\},
$$

a contradiction to (2) establishing (4).

Finally, denote by $g^{\prime} \geqslant 1$ the minimum of $\operatorname{gen}_{C}$ over the nonvoid set $\mathrm{L}_{\mathrm{sc}}(C) \cap M_{1} \cap M_{2}$. Again, let $x^{\prime}$ maximize $\sigma_{1} x_{1}^{\prime}$ over $\mathrm{L}_{\mathrm{sc}}^{\left(g^{\prime}\right)}(C) \cap M_{1} \cap M_{2}$ and suppose $x^{\prime} \in \mathrm{L}_{\mathrm{sc}}^{(1)}\left(\left\{y^{\prime}, z^{\prime}\right\}\right)$ for $y^{\prime}, z^{\prime} \in \mathrm{L}_{\mathrm{sc}}^{\left(g^{\prime}-1\right)}(C)$. As before, we infer that $y_{1}^{\prime}=z_{1}^{\prime}=x_{1}^{\prime},\left|y_{2}^{\prime}\right|,\left|z_{2}^{\prime}\right|>\varepsilon$ and $y_{2}^{\prime} z_{2}^{\prime}<0$. So

$$
\mathrm{L}_{\mathrm{sc}}(C) \cap M_{2}^{+} \supset\left[y^{\prime}, z^{\prime}\right] \cap M_{2}^{+} \neq \emptyset,
$$

which together with (4) finishes the proof.

Example 2.4 (Bi-convexity in $\mathbb{R}^{2} \times \mathbb{R}$ and $2 \times 3$ matrices). - A set $A \subset \mathbb{R}^{k} \times \mathbb{R}^{l}$ is biconvex [1] if the sections $A_{x}, A^{y}$ are convex for every $x \in \mathbb{R}^{k}$ and $y \in \mathbb{R}^{l}$. The bi-convex hull $\mathrm{L}_{(k, l)}(A)$ is defined accordingly. Obviously, $A$ is bi-convex if and only if the set

$$
\begin{gathered}
\left\{\left(\begin{array}{cccccccc}
x_{1} & x_{2} & \ldots & x_{k} & 0 & 0 & \ldots & 0 \\
0 & 0 & \ldots & 0 & y_{1} & y_{2} & \ldots & y_{l}
\end{array}\right) \in \mathbb{M}^{2 \times(k+l)}:\right. \\
\left.\left(x_{1}, x_{2}, \ldots, x_{k} ; y_{1}, y_{2}, \ldots, y_{l}\right) \in A\right\}
\end{gathered}
$$

is lamination convex. Aumann and Hart [1] constructed a compact set $K \subset \mathbb{R}^{2} \times \mathbb{R}^{2}$ such that $\mathrm{L}_{(2,2)}(K)$ is not compact. We will show that this is possible in $\mathbb{R}^{2} \times \mathbb{R}$ and hence also for matrices of the form $\left(\begin{array}{ccc}a & b & 0 \\ 0 & 0 & c\end{array}\right)$.

Let $v_{1}=(0,2), v_{2}=(-1,0), v_{3}=(1,-1), v_{4}=(2,1)$ be the usual four-point configuration. Let $w_{1}=(0,1), w_{2}=(0,0), w_{3}=(1,0), w_{4}=(1,1)$ and

$$
\begin{aligned}
L_{0}= & ([0,1] \times[0,1]) \cup(\{0\} \times[0,2]) \cup([-1,1] \times\{0\}) \cup(\{1\} \times[-1,1]) \\
& \cup([0,2] \times\{1\})=\mathrm{L}_{\mathrm{sc}}\left(\left\{v_{i}, w_{i}\right\}\right) .
\end{aligned}
$$

Finally, let $\tilde{K}=\mathcal{I}\left(\left([0,1] \times\left\{v_{1}, v_{2}, v_{3}, v_{4}\right\}\right) \cup\left(\{1\} \times\left\{w_{1}, w_{2}, w_{3}, w_{4}\right\}\right)\right)$ and $L=$ $\mathcal{I}\left(\left((0,1] \times L_{0}\right) \cup\left(\{0\} \times\left\{v_{1}, v_{2}, v_{3}, v_{4}\right\}\right)\right)$, where $\mathcal{I}(x ; y, z)=(x, y ; z)$ identifies $\mathbb{R} \times \mathbb{R}^{2}$ with $\mathbb{R}^{2} \times \mathbb{R}$. We claim that $\mathrm{L}_{(2,1)}(\tilde{K})=L$ and this is not compact.

Let $w_{i}(t)=\mathcal{I}\left(t, w_{i}\right), v_{i}(t)=\mathcal{I}\left(t, v_{i}\right)$. We have $w_{i}(1) \in \tilde{K}$ and then inductively $w_{i}\left(2^{-k}\right) \in \mathrm{L}_{(2,1)}(\tilde{K})$ for every $i \in\{4,3,2,1\}$ and $k \in \mathbb{N}$, because the following convex combinations are compatible with the definition of bi-convexity: $w_{4}(t / 2)=\frac{1}{2} w_{1}(t)+$ $\frac{1}{2} v_{4}(0)$ and $w_{i}(t)=\frac{1}{2} w_{i+1}(t)+\frac{1}{2} v_{i}(t)$ for $i=3,2,1$. Now it is easy to see that $w_{i}(t) \in \mathrm{L}_{(2,1)}(\tilde{K})$ for every $t \in(0,1]$ and hence $L \subset \mathrm{L}_{(2,1)}(\tilde{K})$. On the other hand, $L$ is bi-convex, so that $\mathrm{L}_{(2,1)}(\tilde{K}) \subset L$. 


\section{The proof of Theorem 1.1}

Notation. - For $\alpha \in\left[0, \frac{\pi}{2}\right]$ let $e_{i}(\alpha)=\left(\sin \alpha+\cos \alpha,(-1)^{i} \sin \alpha, \alpha+1\right)$ and $\gamma(\alpha)=$ $(\sin \alpha, 0, \alpha)$. Let $E_{0}=\left\{e_{i}(\alpha): \alpha \in\left[0, \frac{\pi}{2}\right], i=1,2\right\}$.

LEMMA 3.1. - For every $0<\alpha_{2}<\alpha_{1}<\frac{\pi}{2}, \gamma\left(\alpha_{1}\right) \in \overline{\mathrm{L}\left(E_{0} \cup\left\{\gamma\left(\alpha_{2}\right)\right\}\right)}$.

Proof. - For $i=1,2$, let $\Phi_{i}: \mathbb{R}^{3} \times \mathbb{R}^{3} \rightarrow \mathbb{R}^{3}$ be defined by

$$
\begin{aligned}
\Phi_{i}((a, b, c),(x, y, z))= & \left((a-x)^{2}+(b-y)^{2}-(c-z)^{2},\right. \\
& \sin (z-1)+\cos (z-1)-x, \\
& \left.(-1)^{i} \sin (z-1)-y\right) .
\end{aligned}
$$

For every $\alpha \in\left[\alpha_{2}, \alpha_{1}\right]$ and $i=1,2$ we have $\Phi_{i}\left(\gamma(\alpha), e_{i}(\alpha)\right)=0$, as well as $\operatorname{det}\left(\frac{\partial \Phi_{i}}{\partial(x, y, z)}\left(\gamma(\alpha), e_{i}(\alpha)\right)\right)=2 \cos ^{2} \alpha-2 \neq 0$. By the implicit function theorem, there is $\delta_{0}>0$ and two smooth functions $\varphi_{1}, \varphi_{2}: \mathcal{U}_{\delta_{0}} \rightarrow R^{3}$ defined on the $\delta_{0}$-neighborhood $\mathcal{U}_{\delta_{0}}$ of $\left\{\gamma(\alpha): \alpha \in\left[\alpha_{2}, \alpha_{1}\right]\right\}$ such that $\Phi_{i}\left(w, \varphi_{i}(w)\right)=0$ for $w \in \mathcal{U}_{\delta_{0}}$ and $\varphi_{i}(\gamma(\alpha))=e_{i}(\alpha)$ for $\alpha \in\left[\alpha_{2}, \alpha_{1}\right]$. Note that by the definition of $\Phi_{i}, \varphi_{i}(w)-w$ is a rank-one direction and $\varphi_{i}(w)=e_{i}(\alpha)$ for all $w \in \mathcal{U}_{\delta_{0}}$, where $\alpha+1$ is the third coordinate of $\varphi_{i}(w)$. By making $\delta_{0}$ smaller, we may ensure that $\varphi_{i}(w) \in E_{0}$ for $w \in \mathcal{U}_{\delta_{0}}$. Let $u_{i}(w)=\varphi_{i}(w)-w$. Replacing $\delta_{0}$ by a smaller number again, there is $K>0$ such that the functions $u_{1}, u_{2}$ are $K$-Lipschitz on $\mathcal{U}_{\delta_{0}}$ and $\left\|u_{1}(w)\right\|,\left\|u_{2}(w)\right\| \leqslant K$ for $w \in \mathcal{U}_{\delta_{0}}$.

It is easy to check that $\gamma$ satisfies the equation

$$
\dot{\gamma}(\alpha)=\frac{u_{1}(\gamma(\alpha))+u_{2}(\gamma(\alpha))}{2} .
$$

Next, we will approximate the solution $\gamma$ by a piecewise linear curve with derivatives given by $u_{1}$ on odd and by $u_{2}$ on even segments. We do an easy error estimate usual in numerical analysis.

Let $\delta>0$ be given. Find $n \in \mathbb{N}$ such that, for $h=\left(\alpha_{1}-\alpha_{2}\right) / n, K h<\delta_{0}$ and

$$
\frac{h}{2}(2 \operatorname{Lip} \gamma+K)\left((1+h K)^{n}-1\right)<\min \left(\delta, \frac{\delta_{0}}{2}\right) .
$$

For $k=1, \ldots, n$, define

$$
w_{0}=\gamma\left(\alpha_{2}\right), \quad w_{k-\frac{1}{2}}=w_{k-1}+\frac{h}{2} u_{1}\left(w_{k-1}\right), \quad w_{k}=w_{k-\frac{1}{2}}+\frac{h}{2} u_{2}\left(w_{k-\frac{1}{2}}\right) .
$$

Let $\varepsilon_{k}=\left\|w_{k}-\gamma\left(\alpha_{2}+k h\right)\right\|, k=0,1, \ldots, n$. Then

$$
\begin{aligned}
\varepsilon_{k+1} & =\left\|w_{k+1}-\gamma\left(\alpha_{2}+(k+1) h\right)\right\| \\
& =\left\|w_{k}-\gamma\left(\alpha_{2}+k h\right)+\int_{\alpha_{2}+k h}^{\alpha_{2}+(k+1) h} \frac{u_{1}\left(w_{k}\right)+u_{2}\left(w_{k+\frac{1}{2}}\right)}{2}-\dot{\gamma}(\alpha) \mathrm{d} \alpha\right\|
\end{aligned}
$$

and hence, by (5), 


$$
\begin{aligned}
\varepsilon_{k+1} & \leqslant \varepsilon_{k}+\frac{1}{2} \int_{\alpha_{2}+k h}^{\alpha_{2}+(k+1) h}\left\|u_{1}\left(w_{k}\right)-u_{1}(\gamma(\alpha))\right\|+\left\|u_{2}\left(w_{k+\frac{1}{2}}\right)-u_{2}(\gamma(\alpha))\right\| \mathrm{d} \alpha \\
& \leqslant \varepsilon_{k}+\frac{h}{2}\left(\operatorname{Lip} u_{1}\left(h \operatorname{Lip} \gamma+\varepsilon_{k}\right)+\operatorname{Lip} u_{2}\left(h \operatorname{Lip} \gamma+h\left\|u_{1}\left(w_{k}\right)\right\|+\varepsilon_{k}\right)\right) \\
& \leqslant A \varepsilon_{k}+B
\end{aligned}
$$

where $A=(1+h K)$ and $B=\frac{h^{2} K}{2}(2 \operatorname{Lip} \gamma+K)$. We have $\varepsilon_{0}=0$ and, by induction,

$$
\begin{aligned}
\varepsilon_{k} \leqslant B\left(1+A+A^{2}+\cdots+A^{k-1}\right) & =B\left(A^{k}-1\right) /(A-1) \\
& =\frac{h}{2}(2 \operatorname{Lip} \gamma+K)\left((1+h K)^{k}-1\right) \\
& <\min \left(\delta, \delta_{0} / 2\right) .
\end{aligned}
$$

Hence $w_{k}, w_{k+\frac{1}{2}} \in \mathcal{U}_{\delta_{0}}$ (so that the sequence is well defined) and $\left\|\gamma\left(\alpha_{1}\right)-w_{n}\right\|<\delta$.

Furthermore, $w_{k+\frac{1}{2}} \in\left[w_{k}, \varphi_{1}\left(w_{k}\right)\right], w_{k+1} \in\left[w_{k+\frac{1}{2}}, \varphi_{2}\left(w_{k+\frac{1}{2}}\right)\right]$ and the two segments have rank-one directions, so that $w_{0}, w_{\frac{1}{2}}, \ldots, w_{n}$ belong to the lamination convex hull of $E_{0} \cup\left\{w_{0}\right\}=E_{0} \cup\left\{\gamma\left(\alpha_{2}\right)\right\}$. Since $\delta>0$ was arbitrarily small, $\gamma\left(\alpha_{1}\right)$ lies in its closure.

Remark 3.2. - Under the assumption of Lemma 3.1 we have that $\gamma\left(\alpha_{1}\right)$ belongs to the rank-one convex hull of $E_{0} \cup\left\{\gamma\left(\alpha_{2}\right)\right\}$. Also, the corresponding laminate $\mu$ with barycentre in $\gamma\left(\alpha_{1}\right)$ can be given explicitly:

$$
\mu(A)=\mathrm{e}^{-\left(\alpha_{1}-\alpha_{2}\right)} \delta_{\gamma\left(\alpha_{2}\right)}(A)+\frac{1}{2} \sum_{i=1}^{2} \int_{\left(\alpha_{2}, \alpha_{1}\right) \cap \mathrm{e}_{i}^{-1}(A)} \mathrm{e}^{-\left(\alpha_{1}-\alpha\right)} \mathrm{d} \alpha,
$$

where $\delta_{\gamma\left(\alpha_{2}\right)}$ is the Dirac measure at $\gamma\left(\alpha_{2}\right)$.

Indeed, (6) determinates prelaminate $\mu_{n}$ with barycentre $w_{n}^{(n)}$ supported by finite set $\left\{\gamma\left(\alpha_{2}\right) ; \varphi_{1}\left(w_{k-1}^{(n)}\right), \varphi_{2}\left(w_{k-\frac{1}{2}}^{(n)}\right), k=1, \ldots, n\right\} \subset K$, recall that $u_{i}(w)=\varphi_{i}(w)-w$ is a rank-one direction. We added indices $(n)$ to emphasize that points $w_{s}$ depend on $n$ as well. A calculation shows that the weak limit of $\mu_{n}$ is $\mu$; the barycentre of $\mu$ is $\lim w_{n}^{(n)}=\gamma\left(\alpha_{1}\right)$.

Notation. - Let

$$
\begin{aligned}
& x(\alpha, t)=\sin \alpha+t \cos \alpha, \\
& y(\alpha, t)=t \sin \alpha, \\
& z(\alpha, t)=\alpha+t, \\
& \varphi(\alpha, t)=(x(\alpha, t), z(\alpha, t)) .
\end{aligned}
$$

Also let $P=\left[0, \frac{\pi}{2}\right] \times[0,1]$ and $D=\varphi(P)=\left\{(x, z): z \in\left[0, \frac{\pi}{2}\right], \quad \sin z \leqslant x \leqslant\right.$ $\min (1, z)\} \cup\left\{(x, z): z \in\left[1,1+\frac{\pi}{2}\right], 1 \leqslant x \leqslant \sqrt{2} \sin \left(z+\frac{\pi}{4}-1\right)\right\}$. The function $Y: D \rightarrow$ $[0, \infty)$ is going to be defined by

$$
Y(\varphi(\alpha, t))=y(\alpha, t) \quad(\alpha, t) \in P .
$$


LEMMA 3.3. - Let $\alpha_{1}, \alpha_{2} \in\left[0, \frac{\pi}{2}\right]$ and $\alpha_{1} \neq \alpha_{2}$. Then $\varphi\left(\alpha_{1}, t_{1}\right)=\varphi\left(\alpha_{2}, t_{2}\right)$ if and only if

$$
\begin{aligned}
& t_{1}=t_{1}\left(\alpha_{1}, \alpha_{2}\right)=\frac{\sin \alpha_{1}-\sin \alpha_{2}-\left(\alpha_{1}-\alpha_{2}\right) \cos \alpha_{2}}{\cos \alpha_{2}-\cos \alpha_{1}} \\
& t_{2}=t_{2}\left(\alpha_{1}, \alpha_{2}\right)=\frac{\sin \alpha_{1}-\sin \alpha_{2}-\left(\alpha_{1}-\alpha_{2}\right) \cos \alpha_{1}}{\cos \alpha_{2}-\cos \alpha_{1}}
\end{aligned}
$$

If $\alpha_{1}>\alpha_{2}$ then $t_{1}<0$ and $t_{2}>0$.

Proof. - Formulae (9) are obvious. Assume $\alpha_{1}>\alpha_{2}$. Let $f(x)=\sin x-\sin \alpha_{2}-$ $\left(x-\alpha_{2}\right) \cos \alpha_{2}$. Then $f\left(\alpha_{2}\right)=0$ and $f^{\prime}(x)=\cos x-\cos \alpha_{2}<0$ for $\alpha_{2}<x \leqslant \frac{\pi}{2}$, hence $f\left(\alpha_{1}\right)<0$ and $t_{1}=f\left(\alpha_{1}\right) /\left(\cos \alpha_{2}-\cos \alpha_{1}\right)<0$. Similarly, for $g(x)=\sin \alpha_{1}-\sin x-$ $\left(\alpha_{1}-x\right) \cos \alpha_{1}$ we have $g\left(\alpha_{1}\right)=0$ and $g^{\prime}(x)=-\cos x+\cos \alpha_{1}<0$ for $0 \leqslant x<\alpha_{1}$. Thus $g\left(\alpha_{2}\right)>0$ and $t_{2}>0$.

LEMMA 3.4. - Let the function $t_{2}$ be defined by formula (9) for $\alpha_{2}<\alpha_{1}$ and by $t_{2}\left(\alpha_{1}, \alpha_{2}\right)=0$ if $\alpha_{1}=\alpha_{2}$. Let $\alpha_{1} \in\left(0, \frac{\pi}{2}\right]$ be fixed. Then

$$
D_{\alpha_{1}}=\left\{\varphi\left(\alpha_{2}, t\right): \alpha_{2} \in\left[0, \alpha_{1}\right], t \in\left[t_{2}\left(\alpha_{1}, \alpha_{2}\right), 1\right]\right\}
$$

is a convex subset of $D$.

Proof. - It is easily seen that

$$
\chi(z)= \begin{cases}z, & z \in[0,1], \\ \sqrt{2} \sin \left(z+\frac{\pi}{4}-1\right), & z \in\left[1,1+\frac{\pi}{2}\right]\end{cases}
$$

is a concave function on $I=\left[0,1+\frac{\pi}{2}\right]$ and that $D_{\alpha_{1}}$ is the part of its subgraph $\{(x, z): z \in$ $I, x \leqslant \chi(z)\}$ which lies above the segment $\left\{\varphi\left(\alpha_{1}, t_{1}\right): t_{1} \in\left[t_{1}\left(\alpha_{1}, 0\right), 0\right] \cup[0,1]\right\}=$ $\left\{\varphi\left(\alpha_{2}, t_{2}\left(\alpha_{1}, \alpha_{2}\right)\right): \alpha_{2} \in\left[0, \alpha_{1}\right]\right\} \cup\left\{\varphi\left(\alpha_{1}, t\right): t \in[0,1]\right\}$. (Recall that the functions $t_{1}, t_{2}$ came from $\varphi\left(\alpha_{1}, t_{1}\right)=\varphi\left(\alpha_{2}, t_{2}\right)$.)

LEMmA 3.5. - The function $Y: D \rightarrow[0, \infty)$ is well defined by $(8) . Y$ is a $\mathcal{C}^{\infty}$-smooth function on the interior of $D$.

Proof. - By Lemma 3.3, $\varphi: P \rightarrow D$ is a bijection. The Jacobi determinant of $\varphi$ is $-t \sin \alpha \neq 0$ on int $P$, so that $\varphi$ is a $\mathcal{C}^{\infty}$-diffeomorphism of int $P$ onto int $D$.

Definition 3.6. - Let $T=\{(x, y, z):(x, z) \in D,|y| \leqslant Y(x, z)\}$ and let $F_{i}(\alpha, t)=$ $\left(x(\alpha, t),(-1)^{i} y(\alpha, t), z(\alpha, t)\right)$ so that $F_{2}(P)$ is the "front" surface of $T$. Assume $(\alpha, t) \in$ int $P, S=F_{2}(\alpha, t)$ and $v=A \partial_{\alpha} F_{2}(\alpha, t)+B \partial_{t} F_{2}(\alpha, t)$ where $(A, B) \neq(0,0)$. The line $L=S+\mathbb{R} v$ will be called a tangent at the point $S$. It is said to be an outer or inner or surface tangent if there is $\varepsilon>0$ such that, for every $r \in(-\varepsilon, 0) \cup(0, \varepsilon), S+r v \notin T$ or $S+r v \in T$ or $S+r v \in F_{2}(P)$, respectively. Tangent $L$ is said to be rank-one if $v$ is a rank-one direction. The same terminology will be used for any segment $L=S+\left[r_{1}, r_{2}\right] v$, $r_{1}<0<r_{2}$.

Remark. - In order to give an interpretation of what follows, let us recall that if $\tilde{Y}: \tilde{D} \rightarrow \mathbb{R}$ is a function which has the second differential $\mathrm{D}^{2} \tilde{Y}$ negatively semi-definite 
everywhere on a convex set $\tilde{D}$, then the set $\tilde{T}=\{(x, y, z):(x, z) \in \tilde{D},|y| \leqslant \tilde{Y}(x, z)\}$ is convex.

In our case, $\mathrm{D}^{2} Y$ is "negatively semi-definite with respect to a set of directions" (see Lemma 3.7) and we are going to prove that $T$ is lamination convex (Proposition 3.11). Note that the set of directions is defined in terms of all variables including the dependent one and therefore it depends on the gradient of $Y$. Lemma 3.9 says that $D$ is "sufficiently convex" (which is a property of the pair $D, Y$ ).

LEMMA 3.7. - With the above notation, assume $L$ is a rank-one tangent. Then either it is an outer tangent, or it is a surface tangent with the direction $v=\partial_{t} F_{2}(\alpha, t)$.

Proof. - Let

$$
\begin{aligned}
& u_{1}=\partial_{\alpha} F_{2}(\alpha, t)=(\cos \alpha-t \sin \alpha, t \cos \alpha, 1), \\
& u_{2}=\partial_{t} F_{2}(\alpha, t)=(\cos \alpha, \sin \alpha, 1)
\end{aligned}
$$

A simple calculation shows that $v=A u_{1}+B u_{2}$ is a rank-one direction if and only if

$$
(A, B)=k\left(2 \sin ^{2} \alpha, t^{2}-\sin ^{2} \alpha-2 t \cos \alpha \sin \alpha\right) \quad(k \in \mathbb{R})
$$

or $A=0$. In the second case, $v$ is a multiple of $u_{2}$ and $L$ is a surface tangent because $F_{2}(\alpha, t)$ is a linear function of $t$.

Assume (10) holds true. Let us write $\mathrm{D} f$ and $\mathrm{D}^{2} f$ for the first and second differential of a function $f$ at the point $S_{0}=\varphi(\alpha, t)$, respectively. ( $\mathrm{D}^{2} f$ is a quadratic form.) We will write $\mathrm{D} f(w)=\langle\mathrm{D} f, w\rangle$ and $\mathrm{D}^{2} f(w)$ when they are applied to a direction $w$. The set $F_{2}(P)$ can be viewed as the graph of the function $Y$ (with interchanged second and third coordinates) and $T$ is contained in the subgraph. To show that the tangent $L$ is outer it is enough to verify that the second derivative of $Y$ at $S_{0}$ in the direction $v_{0}=A \partial_{\alpha} \varphi\left(S_{0}\right)+B \partial_{t} \varphi\left(S_{0}\right)$ equals

$$
\mathrm{D}^{2} Y\left(v_{0}\right)=-8 k^{2} \sin ^{4} \alpha \cos \alpha<0 .
$$

Although this could be done directly, we suggest the following way which reduces the size of expressions involved. Let $\omega(s)=\varphi(\alpha+A s, t+B s)$. Then

$$
\left.\frac{\partial^{2}}{\partial s^{2}} Y(\omega(s))\right|_{s=0}=\mathrm{D}^{2} Y\left(v_{0}\right)+\left\langle\mathrm{D} Y,\left(\mathrm{D}^{2} x(A, B), \mathrm{D}^{2} z(A, B)\right)\right\rangle .
$$

On the other hand, $Y(\omega(s))=y(\alpha+A s, t+B s)=(t+B s) \sin (\alpha+A s)$, so that

$$
\left.\frac{\partial^{2}}{\partial s^{2}} Y(\omega(s))\right|_{s=0}=2 A B \cos \alpha-A^{2} t \sin \alpha .
$$

Differentiating (8) and solving the resulting equation we easily obtain

$$
\mathrm{D} Y=\left(\frac{t \cos \alpha-\sin \alpha}{-t \sin \alpha}, \frac{\cos \alpha \sin \alpha-t}{-t \sin \alpha}\right) .
$$


The calculation of $\mathrm{D}^{2} x$ and $\mathrm{D}^{2} z$ is straightforward and gives

$$
\mathrm{D}^{2} x(A, B)=-A^{2}(\sin \alpha+t \cos \alpha)-2 A B \sin \alpha, \quad \mathrm{D}^{2} z(A, B)=0 .
$$

Eqs. (12)-(15) imply

$$
\mathrm{D}^{2} Y\left(v_{0}\right)=-A\left(\frac{A t}{\sin \alpha}-\frac{(A+2 B) \sin \alpha}{t}\right) .
$$

Using (10) we get (11).

LEMMA 3.8. - Let $\left(\alpha_{1}, t_{1}\right) \in P, \alpha_{2} \in\left[0, \frac{\pi}{2}\right]$ and $t_{2}<0$. Let $\varphi\left(\alpha_{1}, t_{1}\right)=\varphi\left(\alpha_{2}, t_{2}\right)$. Then $0 \leqslant y\left(\alpha_{1}, t_{1}\right)<-y\left(\alpha_{2}, t_{2}\right)$.

Proof. - By Lemma 3.3, $\alpha_{1} \leqslant \alpha_{2}$. If $\alpha_{1}=\alpha_{2}$ then $0 \leqslant t_{1}=t_{2}<0$. Thus $\alpha_{1}<\alpha_{2}$ and by (9)

$$
y\left(\alpha_{2}, t_{2}\right)+y\left(\alpha_{1}, t_{1}\right)=\frac{\sin ^{2} \alpha_{2}-\sin ^{2} \alpha_{1}-\left(\alpha_{2}-\alpha_{1}\right) \sin \left(\alpha_{2}+\alpha_{1}\right)}{\cos \alpha_{1}-\cos \alpha_{2}}<0
$$

where the inequality comes from

$$
\begin{aligned}
\left(\alpha_{2}-\alpha_{1}\right) \sin \left(\alpha_{2}+\alpha_{1}\right) & >\sin \left(\alpha_{2}-\alpha_{1}\right) \sin \left(\alpha_{2}+\alpha_{1}\right) \\
& =\frac{1}{2}\left(\cos 2 \alpha_{1}-\cos 2 \alpha_{2}\right) \\
& =\frac{1}{2}\left(1-2 \sin ^{2} \alpha_{1}-1+2 \sin ^{2} \alpha_{2}\right) .
\end{aligned}
$$

Thus $y\left(\alpha_{1}, t_{1}\right)<-y\left(\alpha_{2}, t_{2}\right)$.

LEMMA 3.9. - Let $A=\left(a_{1}, a_{2}, a_{3}\right) \in T$ and $B=\left(b_{1}, b_{2}, b_{3}\right) \in T$ be such that $B-A$ is a rank-one direction. Then $\left[\left(a_{1}, a_{3}\right),\left(b_{1}, b_{3}\right)\right] \subset D$.

Proof. - By assumptions, $A_{0}=\left(a_{1}, a_{3}\right) \in D$ and $B_{0}=\left(b_{1}, b_{3}\right) \in D$, thus there exist $\left(\alpha_{1}, \tau_{1}\right),\left(\alpha_{2}, \tau_{2}\right) \in P$ such that $A_{0}=\varphi\left(\alpha_{1}, \tau_{1}\right), B_{0}=\varphi\left(\alpha_{2}, \tau_{2}\right)$. Furthermore, $\left|a_{2}\right| \leqslant$ $y\left(\alpha_{1}, \tau_{1}\right),\left|b_{2}\right| \leqslant y\left(\alpha_{2}, \tau_{2}\right)$. If $\alpha_{2}=\alpha_{1}$ then obviously $\left[A_{0}, B_{0}\right] \subset D$. We may assume e.g. $\alpha_{2}<\alpha_{1}$.

Let $V_{0}=\left\{(x, y, z): x^{2}+y^{2}-z^{2}<0, z<0\right\}$. $V_{0}$ is an open convex cone. A point $X$ is rank-one connected to $(0,0,0)$ if and only if it belongs to $\partial V_{0}$ when it is below $(0,0,0)$ or $X \in-\partial V_{0}$ when $X$ is above $(0,0,0)$ ("below" and "above" refers to the value of the third coordinate). It is easily seen that if $L$ is a line with rank-one direction and $(0,0,0) \notin L$ then $L$ intersects $\partial V_{0}$ in at most one point and, therefore, $L \cap V_{0}$ is either an open half-line directed "downwards" or empty.

Let $V_{A}=A+V_{0}$ and $V_{1}=\gamma\left(\alpha_{1}\right)+V_{0}$. The point $\gamma\left(\alpha_{1}\right)$ is rank-one connected to $F_{i}\left(\alpha_{1}, \tau_{1}\right)$ and $A \in\left[F_{1}\left(\alpha_{1}, \tau_{1}\right), F_{2}\left(\alpha_{1}, \tau_{1}\right)\right]$ hence $A \in-\overline{V_{0}}+\gamma\left(\alpha_{1}\right), \gamma\left(\alpha_{1}\right) \in \overline{V_{A}}$ and $V_{1} \subset V_{A}$.

Let $t_{1}<0, t_{2}>0$ solve the equation $\varphi\left(\alpha_{1}, t_{1}\right)=\varphi\left(\alpha_{2}, t_{2}\right)$, cf. Lemma 3.3. Since $\gamma\left(\alpha_{1}\right)$ is also rank-one connected to the two points $F_{i}\left(\alpha_{1}, t_{1}\right), i=1,2$, we have $F_{i}\left(\alpha_{1}, t_{1}\right) \in$ $\overline{V_{1}} \subset \overline{V_{A}}$. By Lemma 3.8, with indices 1,2 interchanged, $0 \leqslant y\left(\alpha_{2}, t_{2}\right)<-y\left(\alpha_{1}, t_{1}\right)$. Thus $F_{1}\left(\alpha_{2}, t_{2}\right), F_{2}\left(\alpha_{2}, t_{2}\right)$ are in the open segment $\left(F_{1}\left(\alpha_{1}, t_{1}\right), F_{2}\left(\alpha_{1}, t_{1}\right)\right) \subset V_{A}$. 
Since the direction $\partial_{t} F_{i}\left(\alpha_{1}, t\right)$ of the line $\left\{F_{i}\left(\alpha_{2}, t\right): t \in \mathbb{R}\right\}$ is a rank-one vector directed upwards, we have $F_{i}\left(\alpha_{2}, t\right) \in V_{A}$ for every $t \leqslant t_{2}$. Now, $B \in\left[F_{1}\left(\alpha_{2}, \tau_{2}\right), F_{2}\left(\alpha_{2}, \tau_{2}\right)\right]$ is not in $V_{A}$ since it is rank-one connected to $A$. Therefore $\tau_{2}>t_{2}=t_{2}\left(\alpha_{1}, \alpha_{2}\right)$ and hence $B_{0}=\varphi\left(\alpha_{2}, \tau_{2}\right) \in D_{\alpha_{1}}$.

By Lemma 3.4, it follows that $\left[A_{0}, B_{0}\right] \subset D_{\alpha_{1}} \subset D$.

LemmA 3.10. - Let $A=\left(a_{1}, a_{2}, a_{3}\right) \in T, B=\left(b_{1}, b_{2}, b_{3}\right) \in T, A_{0}=\left(a_{1}, a_{3}\right), B_{0}=$ $\left(b_{1}, b_{3}\right)$. Assume $A$ and $B$ are rank-one connected. Then the open segment $\left(A_{0}, B_{0}\right)$ does not contain any point $\varphi(\alpha, 0), \alpha \in\left[0, \frac{\pi}{2}\right]$. Furthermore $\left(A_{0}, B_{0}\right)$ contains no point $\varphi(0, t), t \in[0,1]$, unless $[A, B] \subset[(0,0,0),(1,0,1)] \subset T$.

Proof. - Let $v=\left(v_{1}, v_{2}, v_{3}\right)=B-A$. Assume there is $\alpha \in\left[0, \frac{\pi}{2}\right]$ such that $S_{0}=$ $\varphi(\alpha, 0) \in\left(A_{0}, B_{0}\right)$. Clearly $\alpha \neq 0$, because $D \subset \mathbb{R} \times \mathbb{R}^{+} \cup\{(0,0)\}$. Since $S_{0}$ is a smooth point of the boundary of $D$ and $\left[A_{0}, B_{0}\right] \subset D$ by Lemma 3.9, we have $\left(v_{1}, v_{3}\right)=$ $k \partial_{\alpha} \varphi(\alpha, 0)=k(\cos \alpha, 1)$ for some $k$. Thus $v_{2}= \pm k \sin \alpha$ because $v$ is assumed to be a rank-one direction. There is no loss of generality in assuming $v_{2}>0$, so that $v=k .(\cos \alpha, \sin \alpha, 1)$.

Note that $v=k \partial_{t} F_{2}(\alpha, 0)$ and $F_{2}$ is linear in $t$. Thus $F_{2}(\alpha, t)=A$ or $F_{2}(\alpha, t)=B$ for some $t<0$. However, Lemma 3.8 immediately implies that $F_{2}(\alpha, t) \notin T$ for every $t<0$ which is a contradiction.

The second assertion is obvious since segment $M=[(0,0),(1,1)]$ is extremal in $D \subset\{(x, z): z \geqslant x\}$ and $Y=0$ on $M$.

Proposition 3.11.- The set $T$ is lamination convex. Any set $\tilde{T}$ such that $T \backslash$ $\left\{\gamma(\alpha): \alpha \in\left(0, \frac{\pi}{2}\right)\right\} \subset \tilde{T} \subset T$ is lamination convex, too.

Proof. - Assume that $T$ is not lamination convex. Then there is $A=\left(a_{1}, a_{2}, a_{3}\right) \in T$, $B=\left(b_{1}, b_{2}, b_{3}\right) \in T$ such that segment $[A, B]$ is not a subset of $T$ and $B-A$ is a rankone direction. We will gradually change the segment with the goal to find an inner tangent parallel to the original $[A, B]$.

Let $A_{0}=\left(a_{1}, a_{3}\right), B_{0}=\left(b_{1}, b_{3}\right)$ and $A_{0}^{\prime}=\left(a_{1}, 0, a_{3}\right), B_{0}^{\prime}=\left(b_{1}, 0, b_{3}\right)$. Obviously $A_{0} \neq B_{0}$. By Lemma 3.9, $\left[A_{0}, B_{0}\right] \subset D$.

We claim that $\left(A_{0}, B_{0}\right) \subset$ int $D$ and thus $\left(A_{0}^{\prime}, B_{0}^{\prime}\right) \subset \operatorname{int} T$. If not, then there is a point $\left(c_{1}, c_{2}, c_{3}\right) \in(A, B)$ such that $\left(c_{1}, c_{2}\right)=\varphi\left(\alpha_{3}, t_{3}\right) \in \partial D$. Hence $\left(\alpha_{3}, t_{3}\right) \in \partial P$. The shape of domain $D$ rules out that $t_{3}=1$. By Lemma 3.10, $t_{3} \neq 0$ and $\alpha_{3} \neq 0$. Thus $\alpha_{3}=\frac{\pi}{2}$ and $a_{1}=b_{1}=c_{1}=1, c_{3} \geqslant \frac{\pi}{2}$. Assume $a_{3} \geqslant c_{3} \geqslant \frac{\pi}{2}$ (otherwise $b_{3} \geqslant c_{3} \geqslant \frac{\pi}{2}$ which is similar). Then $\left|a_{2}\right| \leqslant Y\left(1, a_{3}\right)=y\left(\frac{\pi}{2}, a_{3}-\frac{\pi}{2}\right)=a_{3}-\frac{\pi}{2}$. If $b_{3}<\frac{\pi}{2}$ then, by Lemma 3.8, $\left|b_{2}\right| \leqslant Y\left(1, b_{3}\right)<-y\left(\frac{\pi}{2}, b_{3}-\frac{\pi}{2}\right)=\frac{\pi}{2}-b_{3}$, hence $\left|a_{2}-b_{2}\right| \leqslant\left|a_{2}\right|+\left|b_{2}\right|<a_{3}-b_{3}$ and, in consequence, $A$ and $B$ are not rank-one connected. If $b_{3} \geqslant \frac{\pi}{2}$ then $Y(1, z)=z-\frac{\pi}{2}$ is linear on $\left[b_{3}, a_{3}\right]$ and $[A, B] \subset T$. Since any case leads to a contradiction, we see that, indeed, $\left(A_{0}, B_{0}\right) \subset$ int $D$.

Eventually truncating the segment at a point $(x, 0, z) \in T$, with $(x, z) \in D$, we may assume $a_{2} b_{2} \geqslant 0$. We lose no generality assuming $0 \leqslant a_{2}, 0 \leqslant b_{2}$ because $T$ is symmetric. Finally, we can exchange $A, B$ to have $0 \leqslant a_{2} \leqslant b_{2}$.

Now, we will shift the segment $[A, B]$. For $\tau \in\left[0, b_{2}\right]$, let $A_{\tau}=\left(a_{1}, a_{2}-\tau, a_{3}\right), B_{\tau}=$ $\left(b_{1}, b_{2}-\tau, b_{3}\right)$, and $L_{\tau}=\left[A_{\tau}, B_{\tau}\right] \cap\{(x, y, z): y \geqslant 0\}$. That means $L_{\tau}=\left[\tilde{A}_{\tau}, B_{\tau}\right]$ where 
$\tilde{A}_{\tau}=A_{\tau}$ for $\tau \leqslant a_{2}$ and $\tilde{A}_{\tau} \in\left(A_{0}^{\prime}, B_{0}^{\prime}\right)$ for $a_{2}<\tau<b_{2}$. Recall that $\left(A_{0}^{\prime}, B_{0}^{\prime}\right) \subset \operatorname{int} T$. Let $\operatorname{int}_{D} T$ be the interior of $T$ relative to $\{(x, y, z):(x, z) \in D\}$. For $\tau>0, \tilde{A}_{\tau}, B_{\tau} \in \operatorname{int}_{D} T$.

Let $\tau_{1}=\sup \left\{\tau \in\left[0, b_{2}\right]: L_{\tau} \backslash T \neq \emptyset\right\}$. Obviously $L_{b_{2}} \subset T$ and hence $\tau_{1} \leqslant b_{2}$. Since $T$ is closed we have $L_{\tau_{1}} \subset T$ and $\tau_{1}>0$. Since the endpoints of $L_{\tau_{1}}$ are in $\operatorname{int}_{D} T$, $L_{\tau_{1}}$ must have an interior point $S=\left(s_{1}, s_{2}, s_{3}\right)$ which belongs to the boundary of $T$, i.e. $s_{2}=Y\left(s_{1}, s_{3}\right)$. Since $\left(s_{1}, s_{3}\right) \in \operatorname{int} D$ and $Y$ is a smooth function on int $D, L_{\tau_{1}}$ is a rank-one inner tangent.

By Lemma 3.7, we know that $L_{\tau_{1}}$ must be a surface tangent with the direction $\partial_{t} F_{2}\left(\varphi^{-1}\left(s_{1}, s_{3}\right)\right)$. Since $F_{2}$ is linear in $t, L_{\tau_{1}}$ is in the surface $F_{2}(P)$. However, $\tilde{A}_{\tau_{1}}, B_{\tau_{1}} \in$ $\operatorname{int}_{D} T$. Thus there exists no segment $[A, B]$ as above and $T$ is lamination convex.

As regards points $\gamma(\alpha), \alpha \in\left(0, \frac{\pi}{2}\right)$, the first part of Lemma 3.10 says that they may be freely removed from $T$ and the set remains lamination convex.

Remark. - For $\alpha \in\left(0, \frac{\pi}{2}\right)$, not only the set $T \backslash\{\gamma(\alpha)\}$ is lamination convex. Also for $\hat{T}=T \backslash\left\{F_{i}(\alpha, t): t \in[0,1), i=1,2\right\}$ the same is true. Indeed if $t \in(0,1)$ and $F_{2}(\alpha, t) \in(A, B)$ where the segment $(A, B)$ has rank-one direction and $A, B \in \hat{T}$, then by Lemma 3.7, $(A, B)$ is a surface tangent with the direction $\partial_{t} F_{2}(\alpha, t)$. Hence $A, B$ are in the segment we removed from $T$, a contradiction.

Proof of Theorem 1.1. - Let $0<\alpha_{2}<\alpha_{1}<\frac{\pi}{2}$ and

$$
\begin{aligned}
K & =E_{0} \cup\left\{\gamma\left(\alpha_{2}\right)\right\} \\
& =\left\{\left(\sin \alpha+\cos \alpha,(-1)^{i} \sin \alpha, \alpha+1\right): \alpha \in\left[0, \frac{\pi}{2}\right], i=1,2\right\} \cup\left\{\left(\sin \alpha_{2}, 0, \alpha_{2}\right)\right\} .
\end{aligned}
$$

Then the point $\left(\sin \alpha_{1}, 0, \alpha_{1}\right)$ does not belong to the lamination convex hull of $K$ (Proposition 3.11) but does belong to its closure (Lemma 3.1). For symmetric two by two matrices, the set

$$
\left\{\left(\begin{array}{cc}
z+x & y \\
y & z-x
\end{array}\right):(x, y, z) \in K\right\}
$$

serves as an example.

Remarks. -

(1) It is very easy to see that for every compact set $K, \mathrm{~L}(K)$ is an $F_{\sigma}$-set. Is it always a $G_{\delta}$-set?

(2) We believe that in some classes of compact subsets of $\mathbb{M}_{\text {sym }}^{2 \times 2}$ it is typical, in a sense, for a compact $K$ to have non-closed $\mathrm{L}(K)$. For example if $K$ consists of two curves (or segments) and a point which is rank-one connected to both curves, it is likely that the solution of an equation similar to (5) will move outside $\mathrm{L}(K)$ unless the critical area is covered by other rank-one connections (far from or closely related to the one in (5)). Note, however, that the convex combination coefficients on the right-hand side of (5) have to be properly chosen and, in general, they will depend on $\alpha$. If the above works when the two curves are segments with rank-one directions, $K$ could be replaced by a five-point set.

(3) The first compact $K \subset \mathbb{R}^{3} \cong \mathbb{M}_{\text {sym }}^{2 \times 2}$ for which we had proven non-compactness of $\mathrm{L}(K)$ was 


$$
K=\left\{(x, y, 0): 4(x-1)^{2}+y^{2} \leqslant 4\right\} \cup\left\{\left(a_{0}, 0, \sqrt{8\left(a_{0}-2\right)}\right)\right\},
$$

where $a_{0} \in(2,4]$. The lamination convex superset $T$ of this compact is $\{r((1-$ $\left.t) x+t(4-x), \pm(1-t) \sqrt{4-4(x-1)^{2}}, t \sqrt{8(2-x)}\right): r \in[0,1], t \in[0,1], x \in$ $[0,2]\}$. The method of the proof was quite similar: Contracting a "bad" segment towards point $(0,0,0)$, an inner rank-one tangent would be found, but none exists except "canonical" surface tangents. The sin-based curves in our example were chosen because they lead to much easier calculations at the cost of some additional reasoning.

(4) We do not know whether the set $T$ from Definition 3.6 (considered as a subset of $\mathbb{M}^{2 \times 2}$ ) is rank-one convex or even quasiconvex. Therefore we do not know what are rank-one convex and quasiconvex hulls of $K$. In the case $T$ would be rankone convex, the question Q1 of [2, p. 87 (§ 4.1.2)] would be answered negatively with an impact on understanding of rank-one extreme points.

The set $T$ is not polyconvex. Indeed, taking three matrices $M=\left\{\gamma(0), e_{1}\left(\frac{\pi}{2}\right)\right.$, $\left.e_{2}\left(\frac{\pi}{2}\right)\right\}$ and $t=\left(\frac{\pi^{2}}{2}+2 \pi-2\right) /\left(\pi^{2}+4 \pi\right) \doteq 0.41$, the matrix $X=(1-2 t) \gamma(0)$ $+t e_{1}\left(\frac{\pi}{2}\right)+t e_{2}\left(\frac{\pi}{2}\right)$ belongs to the polyconvex hull of $M$ since the determinants of the three matrices are $d_{0}=0, d_{1}=d_{2}=\frac{\pi^{2}}{4}+\pi-1$ and it is easy to check that determinant of the matrix $X$ equals $(1-2 t) d_{0}+t d_{1}+t d_{2}$. On the other hand, $X \notin T$ since it does not lie "above" $D$. Without giving any details we note that $X$ can be separated from $K$ by a translate of the quasiconvex function $F_{0}$ defined in [5], so that the quasiconvex and polyconvex hulls of $K$ are different.

(5) In a future paper we plan to give another proof of Theorem 1.1 as well as some results related to rank-one convexity, namely a version of Krein-Milman type theorem and the proof that rank-one convex hull and quasiconvex hull in $\mathbb{M}_{\text {sym }}^{2 \times 2}$ have infinite Carathéodory number. Also, we will provide a proof for formula (7) "different" from direct calculation of the limit of corresponding prelaminates.

\section{Upper semi-continuity}

Let $X$ be a metric space. For $\varepsilon>0$, the $\varepsilon$-neighborhood of a set $A \subset X$ will be denoted by $\mathcal{U}_{\varepsilon}(A)=\{x \in X$ : $\operatorname{dist}(x, A)<\varepsilon\}$.

On $\mathcal{K}(X)$, the set of all nonempty compact subsets of $X$, the Hausdorff metric is defined by $\varrho\left(K_{1}, K_{2}\right)=\inf \left\{\varepsilon: K_{1} \subset \mathcal{U}_{\varepsilon}\left(K_{2}\right)\right.$ and $\left.K_{2} \subset \mathcal{U}_{\varepsilon}\left(K_{1}\right)\right\}$. This definition can be extended for non-compact sets $A_{1}, A_{2}$, but it turns out that $\varrho\left(A_{1}, A_{2}\right)=\varrho\left(\bar{A}_{1}, \bar{A}_{2}\right)$.

We say that a function $f: \mathcal{K}(X) \rightarrow \mathcal{K}(X)$ is upper semi-continuous (with respect to Hausdorff metric) if for every $\varepsilon>0$ and $K_{0} \in \mathcal{K}(X)$ there is $\delta>0$ such that $f(K) \subset \mathcal{U}_{\varepsilon}\left(f\left(K_{0}\right)\right)$ whenever $K \in \mathcal{K}(X)$ and $\varrho\left(K, K_{0}\right)<\delta$.

Let $\mathrm{Q}(K)$ denote the quasiconvex hull of a set $K \subset \mathbb{M}^{m \times n}$. In [6], it is shown that the function $K \mapsto \mathrm{Q}(K)$ is upper semi-continuous with respect to Hausdorff metric on the space of all compact subsets of $\mathbb{M}^{m \times n}$. Lamination convex hull and separately lamination convex hull do not share this property.

Proposition 4.1. - Function $K \mapsto \overline{\mathrm{L}_{\mathrm{sc}}(K)}$ is not upper semi-continuous with respect to Hausdorff metric on $\mathcal{K}\left(\mathbb{R}^{3}\right)$. Function $K \mapsto \overline{\mathrm{L}(K)}$ is not upper semi- 
continuous on $\mathcal{K}(X)$ (with respect to Hausdorff metric) where

$$
X=\mathbb{M}_{\text {diag }}^{3 \times 3} \quad \text { or } \quad X=\left\{\left(\begin{array}{lll}
a & b & 0 \\
0 & 0 & c
\end{array}\right)\right\} .
$$

We do not know what the cases of $\mathbb{M}_{\mathrm{sym}}^{2 \times 2}$ and $\mathbb{M}^{2 \times 2}$ look like.

Proof sketch. - Let $K$ be as in (1), $\varepsilon=\frac{1}{3}, J=(0,-1,0), x_{n}=\left(-\frac{1}{2}, \frac{1}{n}, \frac{1}{n}\right) \in \mathrm{L}_{\mathrm{sc}}(K)$, $x=\left(-\frac{1}{2}, 0,0\right) \in \overline{\mathrm{L}_{\mathrm{sc}}(K)} \backslash \mathrm{L}_{\mathrm{sc}}(K), K_{0}=K \cup\{x+J\}, K_{n}=K \cup\left\{x_{n}+J\right\}$. Then $\varrho\left(K_{n}, K_{0}\right) \rightarrow 0$. On the other hand

$$
\begin{aligned}
& \mathrm{L}_{\mathrm{sc}}\left(K_{0}\right) \subset \mathrm{L}_{\mathrm{sc}}(K) \cup[x+J,(0,-1,0)] \quad \text { (a separately lamination convex set) } \\
& \mathrm{L}_{\mathrm{sc}}\left(K_{n}\right) \supset\left[x_{n}, x_{n}+J\right],
\end{aligned}
$$

hence $\mathrm{L}_{\mathrm{sc}}\left(K_{n}\right) \not \subset \mathcal{U}_{\varepsilon}\left(\mathrm{L}_{\mathrm{sc}}\left(K_{0}\right)\right)$. Thus $K \mapsto \overline{\mathrm{L}_{\mathrm{sc}}(K)}$ is not upper semi-continuous on $\mathbb{R}^{3}$ and after a transformation we see that $K \mapsto \overline{\mathrm{L}(K)}$ is not upper semi-continuous on $\mathbb{M}_{\text {diag }}^{3 \times 3}$.

For the last case we start with $\tilde{K}$ and $L$ from Example 2.4 and set $J=(0,0 ;-2)$, $x_{n}=\left(\frac{1}{n}, \frac{1}{2} ; 0\right) \in \mathrm{L}_{(2,1)}(\tilde{K}), x=\left(0, \frac{1}{2} ; 0\right), K_{0}=\tilde{K} \cup\{x+J\}, K_{n}=\tilde{K} \cup\left\{x_{n}+J\right\}$. Again, the segment $\left[x_{n}, x_{n}+J\right]$ is contained in $\mathrm{L}_{(2,1)}\left(K_{n}\right)$ but $[x, x+J]$ does not belong to $\mathrm{L}_{(2,1)}\left(K_{0}\right)$ (nor to its closure) because $K_{0}$ is contained in the bi-convex set $L \cup\{x+J\}$.

Remark. - Let $\mathrm{L}^{\mathrm{c}}(K)$ be the closed lamination convex hull of $K \subset \mathbb{M}^{m \times n}$, i.e., the smallest closed lamination convex set containing $K$. Similarly, the closed separately lamination convex hull $\mathrm{L}_{\mathrm{sc}}^{\mathrm{c}}(K)$ is defined for $K \subset \mathbb{R}^{n}$. There are compacta $K$ such that $\mathrm{L}^{\mathrm{c}}(K) \neq \overline{\mathrm{L}(K)}$ and $\mathrm{L}_{\mathrm{sc}}^{\mathrm{c}}(K) \neq \overline{\mathrm{L}_{\mathrm{sc}}(K)}$, respectively. The two sets named $K_{0}$ above serve as an example. We do not know whether $\mathrm{L}^{\mathrm{c}}(K)=\overline{\mathrm{L}(K)}$ for every compact $K \subset \mathbb{M}_{\text {sym }}^{2 \times 2}$ or $K \subset \mathbb{M}^{2 \times 2}$.

\section{REFERENCES}

[1] R.J. Aumann, S. Hart, Bi-convexity and bi-martingales, Israel J. Math. 54 (1986) 159-180.

[2] B. Kirchheim, Geometry and rigidity of microstructures, Habilitation thesis, Universität Leipzig, 2001.

[3] B. Kirchheim, Private communication.

[4] S. Müller, V. Šverák, Attainment results for the two-well problem by convex integration, in: J. Jost (Ed.), Geometric Analysis and the Calculus of Variations, International Press, Cambridge, MA, 1996, pp. 239-251.

[5] V. Šverák, New examples of quasiconvex functions, Arch. Rat. Mech. Anal. 119 (1992) $293-$ 300.

[6] K. Zhang, On the stability of quasiconvex hulls, Preprint Max-Plank Inst. for Mathematics in the Sciences, Leipzig, 33/1998. 